\title{
VÍDEOS INTERATIVOS COMO FERRAMENTA DE SOCIALIZAÇÃO DE SABERES: VIVÊNCIAS NO USO DE TECNOLOGIAS EDUCACIONAIS NO PROCESSO FORMATIVO
}

\author{
Mayara Evangelista de Andrade (1); Marcelo Costa Fernandes (2) \\ ${ }^{1}$ Enfermeira formada pela Universidade Federal de Campina Grande (UFCG), Estudante de \\ pós-graduação em Enfermagem Obstétrica pela Faculdade Santa Maria (FSM); Membro do \\ Grupo de Pesquisa Laboratório de Tecnologia de Informação e Comunicação em Saúde- \\ LATICS/UFCG/CNPq.E-mail:mayaraeandrade@hotmail.com \\ ${ }^{2}$ Enfermeiro Mestre em Cuidados Clínicos em Saúde pela Universidade Estadual do Ceará \\ (UECE). Doutor pelo programa de Pós-Graduação Cuidados Clínicos em Enfermagem e \\ Saúde pela UECE. Docente da Universidade Federal de Campina Grande (UFCG). Líder do \\ Grupo de Pesquisa Laboratório de Tecnologia de Informação e Comunicação em Saúde- \\ LATICS/UFCG/CNPq. E-mail: celo_cf@hotmail.com
}

\begin{abstract}
RESUMO
A utilização de vídeos interativos como aliado do ensino tem o intuito de aumentar o desenvolvimento socioeducativo provendo melhor acesso à informação e ampliando as possibilidades para os alunos e professores, fazendo com que aprendizagem se torne mais atraente, inovadora, motivadora e significativa. O objetivo desse trabalho é descrever o uso de vídeos interativos como ferramenta de socialização de saberes de acordo com a vivencia no uso de tecnologias educacionais no processo formativo. Trata-se de um relato de experiência de uma participante do projeto de extensão intitulado "Laboratório de tecnologia de informação e comunicação preparando o profissional do amanhã" e do grupo de pesquisa Laboratório de Tecnologias de Informação e Comunicação em Saúde", onde é descrito a experiência na produção de vídeos interativos. O uso de vídeos como ferramenta de socialização de conhecimento é considerado satisfatório e inovador, pois é capaz de motivar, fortalecer e proporcionar maior esclarecimento e aprendizagem dos assuntos expostos, demonstrando eficácia na facilidade e apoio ao processo educativo.
\end{abstract}

PALAVRAS-CHAVE: Tecnologia Educacional; Mídia Audiovisual; saúde.

\section{INTRODUÇÃO}

A utilização de vídeos interativos como aliado do ensino tem o intuito de aumentar o desenvolvimento socioeducativo, provendo melhor acesso à informação e ampliando as possibilidades para os alunos e professores, fazendo com que o processo de aprendizagem se 
torne mais atraente, inovador, motivador e significativo. Essa ferramenta também pode ser utilizada fora das instituições de ensino, corroborando com a transmissão de informações para diversos públicos.

O emprego da tecnologia audiovisual na disseminação de conhecimento torna possível a transformação e o fomento de práticas pedagógicas, dessa forma, os vídeos educativos também podem subsidiar a direção das ações de educação em saúde e estimular a produção de tecnologias educativas dirigidas às demandas das pessoas, famílias e coletividades, com o objetivo de simplificar a aprendizagem e oferecer uma melhoria na qualidade de vida, transmitindo informação para diversos públicos (DALMOLIN et al.,2016, p. 2)

Em pesquisa desenvolvida por Stina, Zamarioli e Carvalho (2015, p. 221), com alunos de graduação em Enfermagem, evidenciaram que o uso de vídeo educativo teve grande relevância na melhoria da percepção cognitiva, portanto, a tecnologia educacional está relacionada à utilização de recursos tecnológicos como instrumento para melhorar o processo ensino e aprendizagem por ser acessível e de rápidos resultados.

Os vídeos interativos, apesar de ser uma ótima ferramenta para mudanças nas metodologias de ensino, são pouco utilizadas e ainda são escassos estudos relacionados a essa temática (SILVA; CARREIRO; MELLO, 2017, p.1050).

O objetivo desse trabalho é descrever o uso de vídeos interativos como ferramenta de socialização de saberes de acordo com a vivencia no uso de tecnologias educacionais no processo formativo.

\section{METODOLOGIA}

Trata-se de um relato de experiência de participantes do projeto de extensão intitulado "Laboratório de tecnologia de informação e comunicação preparando o profissional do amanhã" e do grupo de pesquisa Laboratório de Tecnologias de Informação e Comunicação em Saúde (LATICS) cadastrado no CNPq, onde é relatado a vivência na produção de vídeos interativos.

Este grupo de pesquisa que tem como objetivo proporcionar o empoderamento e a construção de novos conhecimentos críticos e reflexivos no âmbito da saúde, como também divulgar e ampliar os saberes para a sociedade que utiliza da informática como ferramenta de 
pesquisa, por meio da utilização do blog do Laboratório de Tecnologias de Informação e Comunicação em Saúde - LATICS (http://www.laticsufcg.blogspot.com.br/) de caráter educacional e de livre acesso, gravação e disponibilização de videoaulas por meio no canal do LATICS no YouTube (https://www.youtube.com/channel/UCJvYAzFOh9BEP5gt6SB4CmA) e construção de resenhas críticas sobre o contexto da saúde. O grupo visa também a realização de ações de cunho intervencionistas, baseadas na construção de jogos educativos que tratam de assuntos de saúde da comunidade.

\section{DESENVOLVIMENTO}

A produção de vídeos interativos é uma prática dinâmica, que possibilita a socialização rápida de novos saberes, sendo uma oportunidade de compartilhar conhecimento cientifico da área da saúde com inúmeras pessoas.

As temáticas utilizadas nas produções do grupo de pesquisa são todas relacionadas à saúde coletiva, tendo destaque especial a três videoaulas e um curta educativo, todos disponíveis no "YouTube". A primeira a ser publicada foi a aula da "Lei 8.080/90 - Leis orgânicas da saúde", a segunda foi a aula abordando sobre o "Aleitamento Materno" e a ultima sobre "O pacto pela saúde". As visualizações dessas publicações foram de 16.310, 603 e 2138 pessoas, respectivamente.

Os comentários deixados foram positivos em todas aulas, onde os expectadores expressaram as suas opiniões quanto a explanação do assunto, sugestões para outras aulas e relatos sobre experiências na realização de provas de concurso, residências que culminaram na aprovação das pessoas com auxilio dos vídeos.

Nota-se a satisfação das pessoas em assistirem as videoaulas, ao mesmo tempo, é uma atividade que exige muito estudo, muita pesquisa e responsabilidade para que o conteúdo exposto seja abordado de forma séria e com informações verídicas, tendo em vista que essas mídias audiovisuais conseguem atingir milhares de pessoas em diferentes partes do mundo.

O uso de vídeos como ferramenta de socialização de conhecimento é considerado satisfatório e inovador, pois é capaz de motivar, fortalecer e proporcionar um maior esclarecimento e aprendizagem dos assuntos expostos, demonstrando eficácia na facilidade e 
apoio ao processo educativo (DALMOLIN et al., 2016, p. 8), o que torna as tecnologias audiovisuais indispensáveis na formação de novos profissionais.

\title{
CONSIDERAÇÕES FINAIS
}

A utilização dos vídeos interativos como ferramenta de socialização de saberes é percebida como uma tecnologia indispensável no processo formativo. Os vídeos devem ser preparados de forma responsável, com uma linguagem acessível que possa alcançar uma grande diversidade de pessoas, além de contribuir para o processo ensino e aprendizagem durante a formação acadêmica.

É de suma importância ressaltar que as tecnologias educacionais e os vídeos interativos são estratégias que visam contribuir para a capacitação e o empoderamento do público-alvo, inovando os métodos de ensino e aumentando o número de pessoas que a informação consegue alcançar.

\section{INTERACTIVE VIDEOS AS A TOOL OF SOCIALIZATION OF KNOWLEDGE: LIVING IN THE USE OF EDUCATIONAL TECHNOLOGIES IN THE TRAINING PROCESS}

\begin{abstract}
The use of interactive videos as an ally of education aims to increase socio-educational development by providing better access to information and expanding possibilities for students and teachers, making learning more attractive, innovative, motivating and meaningful. The objective of this work is to describe the use of interactive videos as a socialization tool of knowledge according to the experience in the use of educational technologies in the training process. This is an experience report of an extension project participant entitled "Information and communication technology laboratory preparing the professional of tomorrow" and the research group Laboratory of Information and Communication Technologies in Health ", where it is described the Experience in the production of interactive videos. The use of videos as a knowledge socialization tool is considered satisfactory and innovative, since it is able to motivate, strengthen and provide greater clarification and learning of the subjects exposed, demonstrating effectiveness in the ease and support of the educational process.
\end{abstract}

KEYWORDS: Educational Technology; Video-Audio Media; Health. 


\section{VÍDEOS INTERACTIVOS COMO HERRAMIENTA DE SOCIALIZACIÓN DE SABERES: VIVENCIAS EN EL USO DE TECNOLOGÍAS EDUCACIONALES EN EL PROCEDIMIENTO FORMATIVO \\ RESUMEN}

La utilización de vídeos interactivos como aliado de la enseñanza tiene el objetivo de aumentar el desarrollo socioeducativo proporcionando un mejor acceso a la información y ampliando las posibilidades para los alumnos y profesores, haciendo que el aprendizaje se vuelva más atractivo, innovador, motivador y significativo. El objetivo de este trabajo es describir el uso de videos interactivos como herramienta de socialización de saberes de acuerdo con la vivencia en el uso de tecnologías educativas en el proceso formativo. Se trata de un relato de experiencia de una participante del proyecto de extensión titulado "Laboratorio de tecnología de información y comunicación preparando al profesional del mañana" y del grupo de investigación Laboratorio de Tecnologías de Información y Comunicación en Salud ", donde se describe Experiencia en la producción de vídeos interactivos. El uso de vídeos como herramienta de socialización de conocimiento es considerado satisfactorio e innovador, pues es capaz de motivar, fortalecer y proporcionar mayor aclaración y aprendizaje de los asuntos expuestos, demostrando eficacia en la facilidad y apoyo al proceso educativo.

PALABRAS CLAVES: Tecnología Educacional; Medios Audiovisuales; Salud.

\section{REFERÊNCIAS BIBLIOGRÁFICAS}

DALMOLIN, A. et al. Vídeo educativo como recurso para educação em saúde a pessoas com colostomia e familiares. Rev. Gaúcha Enferm., Porto Alegre, v. 37, n. spe, e68373, 2016.

SILVA, D. M. L.; CARREIRO, F. A.; MELLO. R. Tecnologias educacionais na assistência de enfermagem em educação em saúde: revisão integrativa. Rev enferm UFPE on line, Recife, v.11, n.2, p.1044-51, fev, 2017.

STINA, A.P.N.; ZAMARIOLI, C.M.; CARVALHO, E.C. Vídeo educativo e higiene bucal.

Esc. Anna Nery, Ribeirão Preto, v.19, n.2, p.220-225, Abr/Jun, 2015. 